\title{
artigo
}

Sousa, C.N.D.; Araújo, D.A.M.; Sousa, W.G.S.; Lemos, M.H.S.; Rocha, N.R.A.; Negreiros, A.L.B.

Percepção de portadores de doença renal crônica sobre o tratamento hemodialítico

\section{Percepção de portadores de doença renal crônica sobre o tratamento hemodialítico}

\author{
Perception of chronic kidney disease patients about hemodialytic treatment \\ Percepción de los pacientes com enfermedad renal crónica sobre el tratamiento hemodialítico
}

\begin{abstract}
RESUMO
Objetiva-se: Analisar/descrever percepções de portadores de Doença Renal Crônica (DRC) sobre hemodiálise. Método: Revisão integrativa da literatura, com coleta de artigos da base de dados PUBMED durante os dias 09 a 16 de setembro de 2020. Foram analisados 11 artigos, as informações organizadas nas categorias: aspectos físicos versus qualidade de vida; perspectiva de vida; percepção física e suas implicações. Resultado: relativo a aspectos físicos versus qualidade de vida, o paciente com DRC vê-se afetado por aspectos físicos como edema, perda de independência e autonomia nas atividades laborais devido os sintomas. Quanto a perspectiva de vida, observou-se a DRC como limitante ao convívio social e sentença de morte, porém, surgiu como postergadora deste acontecimento. Relativo a percepção física e suas implicações, as mulheres preocupam-se mais com as alterações físicas/estéticas, enquanto os homens preocupam-se com as mudanças de papéis. Conclusão: indica-se o desenvolvimento de intervenções para melhorar o conhecimento sobre a doença.
\end{abstract}

DESCRITORES: Qualidade de Vida; Insuficiência Renal Crônica; Diálise; Hemodiálise.

\section{ABSTRACT}

Objective: Analyze / describe perceptions of hemodialysis patients with Chronic Kidney Disease (CKD). Method: Integrative literature review, with collection of articles from the PUBMED database from September 9 to 16, 2020. 11 articles were analyzed, the information organized in the categories: physical aspects versus quality of life; life perspective; physical perception and its implications. Result: concerning physical aspects versus quality of life, the patient with CKD is affected by physical aspects such as edema, loss of independence and autonomy in work activities due to symptoms. Regarding the perspective of life, CKD was seen as limiting social life and death sentence, however, it appeared as a postponer of this event. Regarding physical perception and its implications, women are more concerned with physical / aesthetic changes, while men are concerned with changing roles. Conclusion: the development of interventions to improve knowledge about the disease is indicated.

DESCRIPTORS: Quality of life; Chronic Kidney Failure; Dialysis; Hemodialysis.

\section{RESUMEN}

Objetivo: Analizar / describir las percepciones de los pacientes en hemodiálisis con Enfermedad Renal Crónica (ERC). Método: Revisión integrativa de la literatura, con recolección de artículos de la base de datos PUBMED del 9 al 16 de septiembre de 2020. Se analizaron 11 artículos, la información organizada en las categorías: aspectos físicos versus calidad de vida; perspectiva de vida; percepción física y sus implicaciones. Resultado: en cuanto a aspectos físicos versus calidad de vida, el paciente con ERC se ve afectado por aspectos físicos como edema, pérdida de independencia y autonomía en las actividades laborales por síntomas. En cuanto a la perspectiva de la vida, la ERC fue vista como una limitación de la vida social y la pena de muerte, sin embargo, apareció como un postponer este hecho. En cuanto a la percepción física y sus implicaciones, las mujeres están más preocupadas por los cambios físicos / estéticos, mientras que los hombres se preocupan por los cambios de roles. Conclusión: se indica el desarrollo de intervenciones para mejorar el conocimiento sobre la enfermedad.

DESCRIPTORES: Calidad de vida; Insuficiencia renal crónica; Diálisis; Hemodiálisis.

RECEBIDO EM: 04/12/2020 APROVADO EM: 01/02/2021

\section{Conceição Nogueira Dias de Sousa}

Enfermeira pela Universidade Federal do Piauí -UFPI. Especialista em saúde do escolar - IFPI.

ORCID: 0000-0002-2455-5532 


\section{Dinah Alencar Melo Araújo}

Enfermeira pela Universidade Federal do Piauí -UFPI, mestranda em ciências e saúde na UFPI. Pós-graduanda em Nefrologia. Pós-graduanda em Urgência e Emergência e UTI.

ORCID: 0000-0003-0922-349X

\section{Waléria Geovana dos Santos Sousa}

Graduanda de enfermagem na Universidade Federal do Piauí -UFPI.

ORCID: 0000-0003-0807-2888

\section{Matheus Henrique da Silva Lemos}

Enfermeiro Intervencionista do SAMU REGIONAL DE COROATÁ-MA. Especialista em Enfermagem em Urgências e Emergências pela UNIFACID-WYDEN. Pós-Graduando em Enfermagem em Saúde do Idoso pelo Instituto Brasileiro de Formação - UNIBF. Mestrando em Ciências e Saúde pela Universidade Federal do Piauí - UFPI.

ORCID: 0000-0002-3554-0141

\section{Naila Roberta Alves Rocha}

Enfermeira atuante no Hospital Regional Deolindo Couto - HRDC em Oeiras-PI e no Instituto Federal do Piauí - Campus Picos. Especialista em Urgência e Emergência e em Terapia Intensiva.

ORCID: 0000-0002-8431-2823

\section{Ana Luiza Barbosa Negreiros}

Enfermeira - Mestra em Ensino na Saúde. Docente de Enfermagem -UFPI.

ORCID: 0000-0002-8313-0403

\section{INTRODUÇÃO}

A Doença Renal Crônica (DRC) é reconhecida mundialmente como um problema de saúde pública. Consiste na perca progressiva e permanente da função dos rins, e está associada ao envelhecimento e à transição demográfica da população. Dentre as principais causas destaca-se a hipertensão arterial e diabetes, no entanto disparidades socioeconômicas, raciais e de gênero também são fatores determinantes. A detecção precoce e o tratamento adequado nos estágios iniciais ajudam a prevenir os desfechos deletérios e a subsequente morbidade relacionados às nefropatias. ${ }^{1}$

As doenças do rim e do trato urinário são responsáveis por aproximadamente 850 milhões de mortes por ano no mundo inteiro e a incidência da DRC aumenta em torno de $8 \%$ ao ano. No Brasil, cerca de 12 milhões de pessoas apresentam algum grau de Insuficiência Renal (IR) e, aproximadamente, 95 mil renais crônicos dependem da hemodiálise (HD) para sobreviverem. ${ }^{2}$

As modalidades disponíveis para o tratamento da DRC com finalidade de substituição parcial das funções renais são: hemodiálise e diálise peritoneal. A
HD pode, muitas vezes, se apresentar como uma esperança de vida para o paciente, já que a doença é irreversível. No entanto, observa-se dificuldades na aceitação do tratamento, da doença e da imagem pessoal. ${ }^{3}$

O paciente com DRC, submetido ao tratamento de hemodiálise, apresenta alterações e restrições físicas, mentais, além de alteração do seu bem-estar, comprometendo sua independência e autonomia. Nesse sentido, provavelmente acontecerão mudanças no comportamento, estilo de vida, hábitos, relações sociais, emprego e renda. ${ }^{4}$

A qualidade de vida dos pacientes com DRC pode ser afetada pela intensidade dos sintomas da doença e por intercorrências clínicas ou complicações paralelas, quantidade de medicação para aliviar os sintomas e alteração da vida social, devido a restrições impostas ao cotidiano pela condição crônica. ${ }^{5}$

Considerando a relevância da HD na vida dos portadores de DRC e a importância da identificação dos impactos que esse tratamento pode causar aos pacientes, faz-se esta análise da literatura sobre as percepções dos portadores de DRC sobre a HD. Objetivando descrever e analisar a percepção do portador de DRC sobre o tratamento hemodialítico, e tendo como questão norteadora: qual(is) a(s) percepção(es) do doente renal crônico sobre o processo de hemodiálise?

\section{MÉTOdO}

Trata-se de uma revisão integrativa da literatura desenvolvida com o intuito de sintetizar achados de pesquisas disponíveis sobre a temática e direcionar a prática fundamentando-se em conhecimento científico. ${ }^{6} \mathrm{O}$ estudo permeou as etapas preconizadas pelo Joanna Briggs Institute: formulação da questão para a elaboração da revisão integrativa da literatura; especificação dos métodos de seleção dos estudos; procedimento de extração dos dados; análise e avaliação dos estudos incluídos na revisão integrativa da literatura; extração dos dados e apresentação da revisão/ síntese do conhecimento produzido e publicado. ${ }^{7}$ Este estudo teve como questão norteadora qual(is) a(s) percepção(es) do doente renal crônico sobre o processo de hemodiálise?

A coleta dos dados deu-se a partir de pesquisa na base de dados PUBMED durante o turno tarde dos dias 09 e 16 


\section{artigo}

Sousa, C.N.D.; Araújo, D.A.M.; Sousa, W.G.S.; Lemos, M.H.S.; Rocha, N.R.A.; Negreiros, A.L.B.

Percepção de portadores de doença renal crônica sobre o tratamento hemodialítico

de setembro de 2020, utilizando os descritores: "percepção", "Insuficiência Renal Crônica" e "Hemodiálise" e o operador booleano and dispondo em três combinações: "percepção and Insuficiência Renal Crônica”; "percepção and Hemodiálise"; "percepção and Insuficiência Renal Crônica and Hemodiálise”.
Com os filtros da base: texto completo, período 2015 a 2020 e formato artigo, obteve-se 140 trabalhos. Em seguida, aplicou-se o critério de inclusão: ser o estudo relacionado a paciente com Doença Renal Crônica em tratamento hemodialítico, excluindo 129 artigos, os quais fugiam da temática.

\section{RESULTADOS}

A amostra da revisão foi composta por 11 estudos, os quais descrevem evidências sobre as percepções dos pacientes com DRC sobre a HD. Os dados extraídos foram compilados na tabela 1 e em seguida discutidos.

\section{Tabela 1: Síntese dos estudos inclusos na revisão integrativa ( $\mathrm{n}=11)$}

Títulos

Living with dying: A narrative inquiry of people with chronic kidney disease and their family members

Illness perception, coping and adherence to treatment among patients with chronic kidney disease

Refusal of Hemodialysis by Hospitalized Chronic Kidney Disease Patients in Pakistan

Qualidade de vida de indivíduos com doença renal crônica em tratamento dialítico

Body Changes and Decreased Sexual Drive after Dialysis: A Qualitative Study on the Experiences of Women at an Ambulatory Dialysis Unit in Spain

Invisible and intangible illness: a qualitative interview study of patients' experiences and understandings of conservatively managed end-stage kidney disease

O corpo marcado pela fístula arteriovenosa: um olhar fenomenológico
Autores

Resultados

Ano
Anita E. Molzahn; Laurene Sheilds; Anne Bruce; Kara Schick-Makaroff; Marcy Antonio; Lacie White2.

\section{Esperanza Velez-Velez; Ricardo J. Bosch}

Salman Tahir Shafi; Mohammad Saleem; Roshina Anjum; Wajid Abdullah; Tahir Shafi.

Nadaby Maria Jesus; Gracielly

Ferreira de Souza; Clesnan Mendes-Rodrigues; Omar Pereira de Almeida Neto; Deusdélia Dias Magalhães Rodrigues; Cristiane Martins Cunha;

Miriam Álvarez-Villarreal; Juan Francisco Velarde-García; Lourdes Chocarro-Gonzalez; Jorge Pérez-Corrales; Javier Gueita-Rodriguez; Domingo Palacios-Ceña.

Katherine Bristowe, Lucy E. Selman, Irene J. Higginson, Fliss E. M. Murtagh.

Dejanilton Melo da Silval; Rose Mary Costa Rosa Andrade Silva; Eliane Ramos Pereiral; Helen Campos Ferreiral; Vanessa Carine Gil de Alcantaral; Fabiana da Silva Oliveira;
O Paciente com DRC e seus familiares destacaram principalmente a relação da finitude da vida e a percepção sobre a morte.

Ser mulher, ter um maior conhecimento da doença e ter um menor senso de controle pessoal afetou a adesão ao tratamento. Aqueles que acreditavam que sua doença tinha um curso crônico eram mais propensos a lidar, isto foi observado como tendência mais forte entre as mulheres.

Entre os que aceitaram a hemodiálise, confiança no conselho do médico foi a razão mais comum. E dentre os que recusaram destaca-se: frequência de HD por semana, natureza vitalícia e permanente, aconselhamento de familiares ou amigos, percepção de baixa qualidade de vida, e medo de agulhas e complicações durante a HD.

As variáveis que mais interferiram na qualidade de vida foram: fumar; fazer hemodiálise (satisfação com a saúde) e tempo das sessões.

Observou-se mudanças corporais como: sendo inchado ou deformado, juntamente com declínio geral. 0 cateter e/ou a fístula desencadeou mudanças na forma de vestir das mulheres. A sexualidade muda, afetando o desejo e a satisfação sexual.

Os participantes descreveram a invisibilidade e intangibilidade da doença renal, e os desafios enfrentados diante os sintomas. Eles descreveram uma presença semelhante a um espectro, minando sua energia e segurando-os.

As alterações causadas pelas Fístulas arteriovenosas provocam baixa autoestima, e atraem o olhar do outro, causando constrangimento naquele que tem o corpo marcado, esse, por sua vez, reage camuflando a fístula. Dessa autocuidado. 
To dialyse or delay: a qualitative study of older New Zealanders' perceptions and experiences of decision-making, with stage 5 chronic kidney disease

Illness Perceptions in Patients on Predialysis Care: Associations With Time Until Start of Dialysis and Decline of Kidney Function

Exploring the nature of illness perceptions in people with end-stage kidney disease

Burden of living with multiple concurrent symptoms in patients with end-stage renal disease

\author{
Sarah Lovell; Robert J Walker; John \\ B.
}

Yvette Meuleman; Moniek C.M. de Goeii; Nynke Halbesma; Joseph Chilcot; Friedo W. Dekker; Sandra van Dijk.

Jessica Rees; Joseph Chilcot; Warren Donnellan; Laura Soulsby;

Marques Shek Nam; Cho Lee Wong; Eva Hau Sim Ho; Yun Ho Hui; Christine Miaskowski; Winnie Kwok Wei So.
Relata a importância de apoio ou presença de algum familiar para a tomada de decisão em dialisar. Também destacou que os participantes viam a diálise como uma maneira de continuarem vivos. Assim como a hemodiálise foi vista como fator de mudanças na rotinas diárias.

As percepções da doença estão associadas a sua progressão, em pacientes com DRC em tratamento pré-dialítico.

O relacionamento encontrado indica que percepções negativas mais fortes da doença no início da pré-diálise especializada pode ser um marcador importante para um início mais precoce da diálise e um declínio mais rápido da função renal.

O potencial para transplante deu aos participantes que realizavam a hemodiálise uma esperança de fuga da diálise.

Emergiu um grupo de sintomas e destacou-se: cansaço, falta de ar, tontura e distúrbios do sono. Também foi descrito três outros temas: "diminuição do funcionamento físico", "mudanças no funcionamento social" e "restrições de dieta e líquidos".

\section{DISCUSSÃO}

A DRC é uma morbidade com repercussões no comportamento individual que, muitas vezes, questiona a existência do sujeito no contexto familiar e nas interações sociais. A realidade configurada na percepção dos sujeitos em HD com Fístula Arteriovenosa - FAV possibilita reconceituar essa nova condição de saúde/doença. Porém, muitas vezes esses sujeitos fazem parte de um mundo de impossibilidades e, ao se depararem com as marcas impostas pela doença em seus corpos, percebem-se e são percebidos como seres diferentes no mundo. Nota-se que a DRC marca a estética corporal de forma contundente, seja pelos sinais da doença, seja pelos acessos invasivos, como a fístula. ${ }^{11}$

É também considerada uma doença silenciosa, invisível e intangível, essa combinação de invisibilidade e intangibilidade, ao lado da natureza imprevisível da doença, impactou fortemente na qualidade de vida dos indivíduos. Além disto, tem a repercussão física, que acaba fazendo que com os pacientes se sintam envergonhados e chateados. Acrescenta-se que as mulheres veem a repercussão física da doença por perspectiva diferenciada, estas reconhecem que a DRC afeta sua sexualidade, diminuindo seu desejo sexual. Algumas associam a diminuição da atividade sexual ao cansaço, especialmente após a HD. Outras se sentem menos atraentes devido às mudanças em sua imagem corporal e, portanto, rejeitam as relações sexuais. ${ }^{8,9,16}$

Indivíduos com DRC têm dificuldade para estabelecer e/ou manter o emprego devido ao tempo que passam em TRS, e sofrem de comprometimento físico e sintomas como fraqueza, cansaço, distúrbios de sono, falta de ar, tontura e mal-estar, que por sua vez afetam o desempenho das atividades da vida diária e produzem efeitos psicológicos e sofrimento emocional. ${ }^{8,10,17}$

Portadores da doença e os familiares muitas vezes têm percepções e desejos muito semelhantes, nomeadamente evitar a dor e minimizar o sofrimento. As pessoas frequentemente compartilham experiências passadas de morte, e a exploração dessas experiências pode facilitar uma compreensão mais profunda dos desejos e medos. ${ }^{12}$ Em alguns momentos os pacientes autodeclaram-se fisicamente incapacitados devido sentirem perda de energia e força. Os diálogos dos pacientes eram semelhantes aos da frase "Carpe Diem" que significa "aproveite o dia”. Este sentido está ligado à mortalidade, no sentindo de en- xergar o diagnóstico como uma "sentença de morte", no entanto, para alguns foi visto como uma oportunidade de mudar as atividades diárias. Os participantes também comentaram que não tinham medo de morrer, mas não queriam sofrer. Muitos, em sua maioria, cientes da expectativa de vida limitada associada à doença, mantem esperança de melhorar e um compromisso de viver bem enquanto viver. ${ }^{12,15}$

Observou-se ainda, que dependendo da idade dos pacientes surge a dicotomia na escolha em dialisar ou não. Em um estudo com 17 idosos em Nova Zelândia os autores observaram que as opiniões e escolhas em dialisar ou não baseou-se em fatos bem individualizados. Os que optaram por não realizar o procedimento, acreditavam que ele iria diminuir a liberdade em estar realizando suas atividades, bem como mencionaram a falta de acompanhantes até a clínica. Os que optaram em fazer afirmaram que o tratamento prolongaria seus anos de vida para aproveitar com a família e até mesmo para ter condições físicas para cuidar de algum membro familiar que estava em estado de saúde mais comprometido. Semelhantes resultados foram observados num estudo do Paquistão envolvendo 125 pacientes. ${ }^{13,14}$

Enquanto para pacientes do sexo mas- 


\section{artigo}

Sousa, C.N.D.; Araújo, D.A.M.; Sousa, W.G.S.; Lemos, M.H.S.; Rocha, N.R.A.; Negreiros, A.L.B.

Percepção de portadores de doença renal crônica sobre o tratamento hemodialítico

culino os conflitos de identidade foram o resultado de uma mudança nos papéis sociais, evidenciado pelas falas que mostraram o sentimento de incapacidade ao levantar objetos pesados como resultado de sua doença, levando-os a tornarem-se dependentes de outras pessoas, sendo difícil assistir esposa/companheira ou algum outro membro familiar assumirem esses deveres. ${ }^{14}$

Como limitações expressas para o desenvolvimento dessa revisão podemos citar a escassez de pesquisas de nível nacional, bem como de níveis de evidência mais fortes, pois devido o tipo de temática, a maioria dos estudos desenvolvidos são de abordagem qualitativa e quantitativa, cujo nível de evidência é VI, além disso, grande parte dos artigos utilizados nessa revisão apresentam amostragens pequenas o que acaba impedindo a realização da generalização dos achados.

\section{CONCLUSÃO}

No aspecto qualidade de vida o paciente com DRC vê-se afetado principalmente por aspectos físicos, perda de independência e autonomia nas atividades laborais diárias devidos. Quanto a perspectiva de vida houve uma dicotomia em perceber a DRC como limitante a até mesmo ao convívio social e sentença de morte, e ao mesmo tempo vista como postergadora deste acontecimento. Levando-os a viver um dia por vez e modificar maneiras de encarar a vida.

Quanto a percepção física foi possível observar maneiras de reação e enfoques diferentes quando tratando-se de sexo masculino e feminino, estas estão mais preocupadas e voltadas para as alterações físicas, enquanto para aqueles estão mais voltados para mudanças de papéis.

Dessa forma, a presente pesquisa demostrou a necessidade do desenvolvimento de intervenções voltadas a disseminação do conhecimento sobre a doença, no intuito de preparar o paciente para lidar com as mudanças induzidas e alterar suas percepções gerais.

\section{REFERÊNCIAS}

1. Marinho AWGB, Penha AP, Silva MT, Galvão TF. Prevalência de doença renal crônica em adultos no Brasil: revisão sistemática da literatura. Cad. Saúde Colet 2017; 25(3): 379-388.

2. Silva RAR, Souza VL, Oliveira GJN, Silva BCO, Rocha CCT, Holanda JRR. Estratégias de enfrentamento utilizadas por pacientes renais crônicos em tratamento hemodialítico. Esc. Anna Nery [online] 2016; 20(1): :147-154.

3. Souto SGT, Lima GS, Silva PLN, Oliveira RS, Gonçalves RPF. Percepção do portador de insuficiência renal crônica quanto às implicações da terapia hemodialítica no seu cotidiano. Rev enferm UERJ 2017; 25: e8093.

4. Bortoluzzi EC, Silva F, Bettinelli LA, Doring M, Fortes VLF, Dobner T.Terapia renal substitutiva: perfil sociodemográfico e clínico laboratorial de pacientes de um serviço de hemodiálise. Rev enferm UFPE on line 2017; 11(9): 3338-45.

5. Jesus NM, Souza GF, Mendes-Rodrigues C, Almeida Neto OP, Rodrigues DDM, Cunha CM. Qualidade de vida de indivíduos com doença renal crônica em tratamento dialítico. Braz J Nephrol 2019; 41(3): 364-374.

6. Souza T, Silva MD, Carvalho R. Revisão integrativa: o que é e como fazer. Einstein 2010; 8(1): 102-6.

7. INSTITUTE TJB. Joanna Briggs Institute Reviewers' Manual: 2014. Edition. [Internet]. Adelaide: Joanna Briggs Institute, 2014. Disponível em: http://joannabriggs.org/assets/docs/sumari/ReviewersManual-2014.pdf. Acesso em: 16 set 2020.

8. Álvarez-Villarreal M, Velarde-García JF, Chocarro-Gonzalez L, Pérez-Corrales J, Gueita-Rodriguez J, Palacios-Ceña D. Body Changes and Decreased Sexual Drive after Dialysis: A Qualitative Study on the Experiences of Women at an Ambulatory Dialysis Unit in Spain. Int. J. Environ. Int J Environ Res Public Health 2019; 16(17): 3086.

9. Bristowe K, Selman LE, Higginson IJ, Murtagh FEM. Invisible and intangible illness: a qualitative interview study of patients' experiences and understandings of conservatively managed end-stage kidney disease. Ann Palliat Med, Abril 2019; 8(2): 121-129.

10. Ng MSN, Wong CL, Ho EHS, Hui YH, Miaskowski C, So WKW. Burden of living with multiple concurrent symptoms in patients with end-stage renal disease. J Clin Nurs 2020.

11. Silva DM, Silva RMCRA, Pereira ER, Ferreira HC, Alcantara VCG, Oliveira FS. O corpo marcado pela fístula arteriovenosa: um olhar fenomenológico. Rev Bras Enferm [Internet] 2018; 71(6): 2869-75.

12. Molzahn $A E$, Sheilds $L$, Bruce $A$, Schick Makaroff $K$, Antonio $M$, White L. Living with dying: A narrative inquiry of people with chronic kidney disease and their family members. J Adv Nurs 2019; 75(1): 129-137.

13. Lovell S,Walker RJ, Schollum JBW, Marshall MR, McNoe BM, Derrett S.To dialyse or delay: a qualitative study of older New Zealanders' perceptions and experiences of decisionmaking, with stage 5 chronic kidney disease. BMJ Open 2017; 7(3): e014781.

14. Shafi ST, Saleem M, Anjum R, Abdullah W, Shafi T. Refusal of Hemodialysis by Hospitalized Chronic Kidney Disease Patients in Pakistan. Saudi J Kidney Dis Transpl [serial online] 2018;29(2):401-408.

15. Jessica Rees J, Chilcot J, Donnellan W, Soulsby L. Exploring the Nature of IIIness Perceptions in People With End-Stage Kidney Disease. J Ren Care 2018; 44(1): 19-29.

16. Vélez-Vélez E, Bosch RJ. Illness perception, coping and adherence to treatment among patients with chronic kidney disease. J Adv Nurs 2016 Apr;72(4):849-63.

17. Pichinelli JJ, Milagres CS. Percepção da insuficiência renal crônica e enfrentamento de pacientes jovens em tratamento hemodialítico. Rev Enfermagem Brasil 2018; 17(3):182-189. 